\title{
A combinatorial identity of multiple zeta values with even arguments*
}

\author{
Shifeng Ding, Lihua Feng, Weijun Liu \\ Department of Mathematics \\ Central South University \\ Changsha 410083, China \\ dingssff@163.com
}

Submitted: Dec 1, 2013; Accepted: Apr 4, 2014; Published: May 9, 2014

Mathematics Subject Classifications: 05A19, 11M06

\begin{abstract}
Let $\zeta\left(s_{1}, s_{2}, \cdots, s_{k} ; \alpha\right)$ be the multiple Hurwitz zeta function. Given two positive integers $k$ and $n$ with $k \leqslant n$, let $E(2 n, k ; \alpha)$ be the sum of all multiple zeta values with even arguments whose weight is $2 n$ and whose depth is $k$. In this note we present some generating series for the numbers $E(2 n, k ; \alpha)$.
\end{abstract}

Keywords: Multiple zeta values; Recursion algorithm; Generating series

\section{Introduction}

The multiple zeta values

$$
\zeta\left(s_{1}, s_{2}, \cdots, s_{m}\right)=\sum_{n_{1}>n_{2}>\cdots>n_{k}>0} \frac{1}{n_{1}^{s_{1}} n_{2}^{s_{2}} \cdots n_{m}^{s_{m}}},
$$

are also called Euler-Zagier sums, where $s_{1}, s_{2}, \cdots, s_{m}$ are positive integers with $s_{1} \geqslant 2$. The multiple Hurwitz zeta function $\zeta\left(s_{1}, s_{2}, \cdots, s_{k} ; \alpha\right)$ is defined by the multiple series

$$
\zeta\left(s_{1}, s_{2}, \cdots, s_{k} ; \alpha\right)=\sum_{n_{1}>n_{2}>\cdots>n_{k} \geqslant 0} \frac{1}{\left(n_{1}+\alpha\right)^{s_{1}}\left(n_{2}+\alpha\right)^{s_{2}} \cdots\left(n_{k}+\alpha\right)^{s_{k}}},
$$

where $\alpha \in \mathbb{C} \backslash\{0,-1,-2, \cdots\}$ and $s_{1}, s_{2}, \cdots, s_{k}$ are positive integers with $s_{1}>1$. The multiple zeta functions have attracted considerable interest in recent years.

*This work was supported by Natural Science Foundation of China (Nos.11101245, 61271355, 11271208, 61202362, 11301302), China Postdoctoral Science Foundation (No.2013M530869), Natural Science Foundation of Shandong (No. BS2013SF009). 
There are various relations among multiple zeta values. One of the well-known Q-linear relations among multiple zeta values is the sum formula ( see $[2,3,4,7,9]$ ), which states that

$$
\sum_{\substack{j_{1}+\cdots+j_{k}=n \\ \operatorname{Each} j_{i} \geqslant 1, j_{1}>1}} \zeta\left(j_{1}, j_{2}, \cdots, j_{k}\right)=\zeta(n) .
$$

In [6], M. Igarashi proved a generalization of the sum formula. Here we quote Igarashi's result in a slightly different form.

Theorem 1. ([6]) Let $\alpha$ be a complex number with $\operatorname{Re} \alpha>0$ and let $n, k$ be positive integers such that $n \geqslant k+1$. Then the following identity holds.

$$
\begin{aligned}
& \sum_{\substack{j_{1}+j_{2}+\cdots+j_{k}=n \\
\operatorname{Each} j_{i} \geqslant 1, j_{1} \geqslant 2}} \zeta\left(j_{1}, j_{2}, \cdots, j_{k} ; \alpha\right) \\
= & \left.\frac{1}{(n-k-1) !} \sum_{N=0}^{\infty} \frac{1}{(N+\alpha)^{k}}\left[\frac{(1-x)_{N}}{(\alpha-x)_{N+1}}\right]^{(n-k-1)}\right|_{x=0},
\end{aligned}
$$

where $[\cdot]^{(n-k-1)}$ denotes the $(n-k-1)$ th derivative of a function with respect to its variable $x$, and $(a)_{N}$ denotes the Pochhammer symbol.

Given two positive integers $n$ and $k$ with $n \geqslant k$, let $E(2 n, k ; \alpha)$ be the sum of all the multiple zeta values of even-integer arguments having weight $2 n$ and depth $k$, i.e.,

$$
E(2 n, k ; \alpha)=\sum_{\substack{j_{1}+\cdots+j_{k}=n \\ j_{1}, j_{2}, \cdots, j_{k} \geqslant 1}} \zeta\left(2 j_{1}, 2 j_{2}, \cdots, 2 j_{k} ; \alpha\right) .
$$

(The notation $E(2 n, k ; \alpha)$ is similar to that in [5, 12]. In [5], Hoffman defined a number $E(2 n, k)$ which is equivalent to the number $E(2 n, k ; 1)$ in our notation, and in [12], the number $E(2 n, k ; 1 / 2) / 2^{2 n}$ in our notation is denoted by $T(2 n, k)$.)

The result of Gangl, Kaneko and Zagier [1] that

$$
E(2 n, 2 ; 1)=\frac{3}{4} \zeta(2 n), \text { for } n \geqslant 2 .
$$

was extended by Shen and Cai [11] to

$$
\begin{gathered}
E(2 n, 3 ; 1)=\frac{5}{8} \zeta(2 n)-\frac{1}{4} \zeta(2) \zeta(2 n-2), \quad \text { for } n \geqslant 3, \\
E(2 n, 4 ; 1)=\frac{35}{64} \zeta(2 n)-\frac{5}{16} \zeta(2) \zeta(2 n-2), \quad \text { for } n \geqslant 4 .
\end{gathered}
$$

Applying the theory of symmetric functions, Hoffman [5] and Zhao [12], respectively, established the generating functions for the numbers $E(2 n, k ; 1)$ and $T(2 n, k)$, respectively. Hoffman [5] proved that

$$
1+\sum_{n \geqslant k \geqslant 1} E(2 n, k ; 1) t^{n} s^{k}=\frac{\sin (\pi \sqrt{1-s} \sqrt{t})}{\sqrt{1-s} \sin (\pi \sqrt{t})} .
$$


And Zhao [12] proved that

$$
1+\sum_{n \geqslant k \geqslant 1} T(2 n, k) t^{n} s^{k}=\frac{\cos (\pi \sqrt{(1-s) t} / 2)}{\cos (\pi \sqrt{t} / 2)} .
$$

Based on these generating functions, some formulas for the numbers $E(2 n, k ; 1)$ and $T(2 n, k)$ for arbitrary $n \geqslant k$ have been obtained. For example,

$$
\begin{aligned}
E(2 n, k ; 1) & =\frac{\zeta(2 n)}{2^{2(k-1)}}\left(\begin{array}{c}
2 k-1 \\
k
\end{array}\right)-\sum_{j=1}^{\left[\frac{k-1}{2}\right]} \frac{\zeta(2 j) \zeta(2 n-2 j)}{2^{2 k-3}(2 j+1) B_{2 j}}\left(\begin{array}{c}
2 k-2 j-1 \\
k
\end{array}\right), \\
T(2 n, k) & =\frac{t(2 n)}{2^{2(k-1)} k}\left(\begin{array}{c}
2 k-2 \\
k-1
\end{array}\right)-\sum_{j=1}^{\left\lfloor\frac{k-1}{2}\right\rfloor} \frac{t(2 j) t(2 n-2 j)}{2^{2 k-3}\left(2^{2 j}-1\right) k B_{2 j}}\left(\begin{array}{c}
2 k-2 j-2 \\
k-1
\end{array}\right),
\end{aligned}
$$

where $B_{2 j}$ is the $2 j$ th Bernoulli number.

In this note we use a method introduced by Granville [2] to establish an algorithm similar to that in Theorem 1 for the calculation of the numbers $E(2 n, k ; \alpha)$. We prove that the calculation of $E(2 n, k ; \alpha)$ involves the Euler $\Gamma$-function and the direct formulas for $E(2 n, k ; 1)$ and $E(2 n, k ; 1 / 2)$ can be deduced from the Bessel functions of the first kind. The main results of this paper are the following theorems.

Theorem 2. Let $\alpha$ be a complex number with $\alpha \in \mathbb{C} \backslash\{0,-1,-2, \cdots\}$. Let $N \geqslant 0$ denote an integer and let $\left(a_{0}^{(N)}, a_{1}^{(N)}, a_{2}^{(N)}, \cdots\right)$ be an infinite series defined by the expansion

$$
\prod_{\substack{r=0 \\ r \neq N}}^{\infty}\left(1+\frac{x}{(r+\alpha)^{2}-(N+\alpha)^{2}}\right)=a_{0}^{(N)}+a_{1}^{(N)} x+\cdots+a_{k-1}^{(N)} x^{k-1}+\cdots .
$$

Then for positive integers $n$ and $k$ (suppose $n \geqslant k$ ), we have

$$
E(2 n, k ; \alpha)=\sum_{N=0}^{\infty} \frac{a_{k-1}^{(N)}}{(N+\alpha)^{2 n-2 k+2}} .
$$

Theorem 3. Given an integer $N \geqslant 0$ and a complex number $\alpha \in \mathbb{C} \backslash\{0,-1,-2, \cdots\}$, for any $x \in \mathbb{C}$ we have

$$
\begin{aligned}
& \prod_{\substack{r=0 \\
r \neq N}}^{\infty}\left(1+\frac{x}{(r+\alpha)^{2}-(N+\alpha)^{2}}\right) \\
= & \frac{2(-1)^{N}(N+\alpha) \Gamma(2 \alpha+N)}{N !} \frac{1}{x \Gamma\left(\alpha+\sqrt{(N+\alpha)^{2}-x}\right) \Gamma\left(\alpha-\sqrt{(N+\alpha)^{2}-x}\right)},
\end{aligned}
$$

where $\Gamma$ denotes Euler's Gamma function. 
Corollary 4. Theorem 2 and Theorem 3 yield that:

$$
\begin{aligned}
& E(2 n, k ; \alpha) \\
= & \left.\frac{2}{k !} \sum_{N=0}^{\infty} \frac{(-1)^{N} \Gamma(N+2 \alpha)}{N !(N+\alpha)^{2 n-2 k+1}}\left[\frac{1}{\Gamma\left(\alpha+\sqrt{(N+\alpha)^{2}-x}\right) \Gamma\left(\alpha-\sqrt{(N+\alpha)^{2}-x}\right)}\right]^{(k)}\right|_{x=0},
\end{aligned}
$$

where $[\cdot]^{(k)}$ denotes the $k$ th derivative of a function with respect to its variable $x$ and $\Gamma$ denotes the Gamma function.

Corollary 5. For positive integers $n$ and $k$ (suppose $n \geqslant k$ ), we have

$$
\begin{aligned}
E(2 n, k ; 1) & =\left.\frac{4(-1)^{k+1} \pi^{2 k}}{k !} \sum_{N=0}^{\infty} \frac{(-1)^{N}}{(N+1)^{2 n-2 k}}(\cos \sqrt{x})^{(k+1)}\right|_{x=(N \pi+\pi)^{2}}, \\
E\left(2 n, k ; \frac{1}{2}\right) & =\left.\frac{2(-1)^{k} \pi^{2 k-1}}{k !} \sum_{N=0}^{\infty} \frac{(-1)^{N}}{\left(N+\frac{1}{2}\right)^{2 n-2 k+1}}(\cos \sqrt{x})^{(k)}\right|_{x=(N \pi+\pi / 2)^{2}} .
\end{aligned}
$$

Corollary 6. The direct formulas for $E(2 n, k ; 1)$ and $E\left(2 n, k ; \frac{1}{2}\right)$ are that:

$$
\begin{aligned}
& E(2 n, k ; 1)=\frac{1}{2^{2 k-2} k !} \sum_{j=0}^{\left\lfloor\frac{k-1}{2}\right\rfloor} \frac{(-1)^{j}(2 k-1-2 j) !(2 \pi)^{2 j}}{(2 j+1) !(k-1-2 j) !} \cdot \zeta(2 n-2 j), \\
& E\left(2 n, k ; \frac{1}{2}\right)=\frac{1}{2^{2 k-2} k !} \sum_{j=0}^{\left\lfloor\frac{k-1}{2}\right\rfloor} \frac{(-1)^{j}(2 k-2-2 j) !(2 \pi)^{2 j}}{(2 j) !(k-1-2 j) !} \zeta\left(2 n-2 j ; \frac{1}{2}\right) .
\end{aligned}
$$

\section{Some Lemmas}

This paper uses some properties of Euler's Gamma function $\Gamma(s)$ and the Bessel function $J_{p}(x)$ of the first kind. Here we list some needed Lemmas.

There are several equivalent forms of the Gamma function $\Gamma(s)$ developed by Euler, one by Weierstrass:

$$
\frac{1}{\Gamma(s)}=s e^{\gamma s} \prod_{m=1}^{\infty}\left(1+\frac{s}{m}\right) e^{-\frac{s}{m}}, \quad \forall s \in \mathbb{C},
$$

where $\gamma$ denotes the Euler-Mascheroni constant defined by

$$
\gamma=\lim _{m \rightarrow \infty}\left(1+\frac{1}{2}+\cdots+\frac{1}{m}-\log m\right)=0.577215 \cdots
$$

It is easy to deduce from Weierstrass' definition of $\Gamma(s)$ the following lemma. 
Lemma 7. Let $\alpha_{1}, \alpha_{2}, \cdots, \alpha_{k}$ and $\beta_{1}, \beta_{2}, \cdots, \beta_{k}$ be two groups of complex numbers $(k \geqslant$ 1). Suppose they satisfy the following conditions: (i) $\alpha_{1}+\alpha_{2}+\cdots+\alpha_{k}=\beta_{1}+\beta_{2}+\cdots+\beta_{k}$ and (ii) none of the $\beta_{j} s$ is a negative integer. Then we have

$$
\prod_{m=1}^{\infty} \frac{\left(m+\alpha_{1}\right)\left(m+\alpha_{2}\right) \cdots\left(m+\alpha_{k}\right)}{\left(m+\beta_{1}\right)\left(m+\beta_{2}\right) \cdots\left(m+\beta_{k}\right)}=\frac{\Gamma\left(1+\beta_{1}\right) \Gamma\left(1+\beta_{2}\right) \cdots \Gamma\left(1+\beta_{k}\right)}{\Gamma\left(1+\alpha_{1}\right) \Gamma\left(1+\alpha_{2}\right) \cdots \Gamma\left(1+\alpha_{k}\right)} .
$$

It is well known that $\Gamma(s)$ satisfies the functional relation $\Gamma(s+1)=s \Gamma(s), \forall s \in$ $\mathbb{C} \backslash\{0,-1,-2, \cdots\}$, and if $N \geqslant 0$ is an integer then $\Gamma(s)$ has residue $\frac{(-1)^{N}}{N !}$ at $s=-N$.

Lemma 8. [10] The following identities hold for $s \in \mathbb{C}$ :

$$
\frac{1}{\Gamma(1+s) \Gamma(1-s)}=\frac{\sin \pi s}{\pi s}, \quad \frac{1}{\Gamma\left(\frac{1}{2}+s\right) \Gamma\left(\frac{1}{2}-s\right)}=\frac{\cos \pi s}{\pi} .
$$

We turn to introduce some properties of the Bessel function of the first kind with a half-integer index. The Bessel function $J_{p}(x)$ is said to be of a half integer index if $p=k \pm \frac{1}{2}$ with $k$ being an integer. It is well known that the Bessel function of the first kind with a half integer index can be represented by elementary functions.

Lemma 9. $\frac{d^{k}}{d x^{k}} \cos (\sqrt{x})=\sqrt{\frac{\pi}{2}}(-1)^{k} 2^{-k} x^{\frac{1-2 k}{4}} J_{k-\frac{1}{2}}(\sqrt{x})$, for $x>0$ and $k=0,1,2, \cdots$.

Proof. The Bessel function $J_{p}(x)$ is defined by the series

$$
J_{p}(x)=\left(\frac{x}{2}\right)^{p} \sum_{m=0}^{\infty} \frac{(-1)^{m}}{m ! \Gamma(p+m+1)}\left(\frac{x}{2}\right)^{2 m},
$$

where the radius of convergence of the series is $+\infty$. It follows from (7) that (using the well-known identity $\left.\Gamma(n+1 / 2)=\sqrt{\pi}(2 n) ! /\left(n ! 2^{2 n}\right), \forall n \geqslant 0\right)$

$$
J_{k-\frac{1}{2}}(\sqrt{x})=\sqrt{\frac{2}{\pi}} 2^{k} x^{\frac{2 k-1}{4}} \sum_{m=0}^{\infty} \frac{(-1)^{m}(m+k) !}{m !(2 m+2 k) !} x^{m} .
$$

On the other hand, by Taylor's expansion of $\cos \sqrt{x}$, we have

$$
\begin{aligned}
\frac{d^{k}}{d x^{k}} \cos (\sqrt{x}) & =\sum_{m=k}^{\infty} \frac{(-1)^{m} m(m-1) \cdots(m-k+1)}{(2 m) !} x^{m-k} \\
& =\sum_{m=0}^{\infty} \frac{(-1)^{m+k}(m+k) !}{(2 m+2 k) ! m !} x^{m}
\end{aligned}
$$

Observing the right-hand sides of (8) and (9), the result follows immediately.

Lemma 10. Let $k \geqslant 0$ be an integer and let $x>0$. Then the Bessel function $J_{k+1 / 2}(x)$ is represented by 


$$
\begin{aligned}
J_{k+\frac{1}{2}}(x)=\sqrt{\frac{2}{\pi}} \frac{1}{\sqrt{x}}\{ & \sin x \sum_{j=0}^{\left\lfloor\frac{k}{2}\right\rfloor} \frac{(-1)^{j}(2 k-2 j) !}{(2 j) !(k-2 j) !} \cdot \frac{1}{(2 x)^{k-2 j}} \\
& \left.\quad-\cos x \sum_{j=0}^{\left\lfloor\frac{k-1}{2}\right\rfloor} \frac{(-1)^{j}(2 k-1-2 j) !}{(2 j+1) !(k-1-2 j) !} \cdot \frac{1}{(2 x)^{k-1-2 j}}\right\} .
\end{aligned}
$$

Proof. We omit the detailed steps. It is shown that ([8]) the Bessel function $J_{k+1 / 2}(x)$ satisfies the relation

$$
J_{k+1 / 2}(x)=(-1)^{k} \sqrt{\frac{2}{\pi x}} x^{k+1} \frac{d^{k}}{(x d x)^{k}}\left(\frac{\sin x}{x}\right), \text { for } k=0,1,2, \cdots .
$$

It follows from (11) that

$$
J_{1 / 2}=\sqrt{\frac{2}{\pi}} \frac{\sin x}{\sqrt{x}}, \quad J_{3 / 2}(x)=\sqrt{\frac{2}{\pi}} \frac{1}{\sqrt{x}}\left(\frac{\sin x}{x}-\cos x\right), \cdots,
$$

and so on. Then using induction on $k$ we can prove that (10) is equivalent to (11).

Lemma 11. Let $k \geqslant 1$ be an integer. Then by Lemma 9 and Lemma 10, we have

$$
\begin{aligned}
(\cos \sqrt{x})^{(k)=} & \frac{(-1)^{k}}{2^{2 k-1}} \frac{\sin \sqrt{x}}{\sqrt{x}} \sum_{j=0}^{\left\lfloor\frac{k-1}{2}\right\rfloor} \frac{(-1)^{j}(2 k-2-2 j) ! 2^{2 j}}{(2 j) !(k-1-2 j) !} \cdot \frac{1}{x^{k-1-j}} \\
& -\frac{(-1)^{k}}{2^{2 k-2}} \cos \sqrt{x} \sum_{j=0}^{\left\lfloor\frac{k-2}{2}\right\rfloor} \frac{(-1)^{j}(2 k-3-2 j) ! 2^{2 j}}{(2 j+1) !(k-2-2 j) !} \cdot \frac{1}{x^{k-1-j}} .
\end{aligned}
$$

\section{Proofs}

Proof of Theorem 2. The left side of (2) is

$$
\begin{aligned}
& \sum_{\substack{j_{1}+\cdots+j_{k}=n \\
j_{1}, \cdots, j_{k} \geqslant 1}} \sum_{n_{1}>n_{2}>\cdots>n_{k} \geqslant 0} \frac{1}{\left(n_{1}+\alpha\right)^{2 j_{1}}\left(n_{2}+\alpha\right)^{2 j_{2}} \cdots\left(n_{k}+\alpha\right)^{2 j_{k}}} \\
& =\sum_{n_{1}>n_{2}>\cdots>n_{k} \geqslant 0} \sum_{\substack{j_{1}+\cdots+j_{k}=n \\
j_{1}, \cdots, j_{k} \geqslant 1}} \frac{1}{\left(n_{1}+\alpha\right)^{2 j_{1}}\left(n_{2}+\alpha\right)^{2 j_{2}} \cdots\left(n_{k}+\alpha\right)^{2 j_{k}}} .
\end{aligned}
$$

The second sum in (13) is the coefficient of $x^{2 n}$ in the formal power series

$$
\sum_{j=1}^{\infty}\left(\frac{x}{n_{1}+\alpha}\right)^{2 j} \sum_{j=1}^{\infty}\left(\frac{x}{n_{2}+\alpha}\right)^{2 j} \cdots \sum_{j=1}^{\infty}\left(\frac{x}{n_{k}+\alpha}\right)^{2 j}
$$




$$
\begin{aligned}
& =\frac{x^{2 k}}{\left[\left(n_{1}+\alpha\right)^{2}-x^{2}\right]\left[\left(n_{2}+\alpha\right)^{2}-x^{2}\right] \cdots\left[\left(n_{k}+\alpha\right)^{2}-x^{2}\right]} \\
& =\sum_{j=1}^{k}\left(\frac{x^{2 k}}{\left(n_{j}+\alpha\right)^{2}-x^{2}} \prod_{\substack{1 \leqslant m \leqslant k \\
m \neq j}} \frac{1}{\left(n_{m}+\alpha\right)^{2}-\left(n_{j}+\alpha\right)^{2}}\right) .
\end{aligned}
$$

It follows that the coefficient of $x^{2 n}$ above is

$$
\sum_{j=1}^{k}\left(\frac{1}{\left(n_{j}+\alpha\right)^{2 n-2 k+2}} \prod_{\substack{1 \leqslant m \leqslant k \\ m \neq j}} \frac{1}{\left(n_{m}+\alpha\right)^{2}-\left(n_{j}+\alpha\right)^{2}}\right) .
$$

Hence the sum (13) is

$$
\sum_{n_{1}>n_{2}>\cdots>n_{k} \geqslant 0} \sum_{j=1}^{k}\left(\frac{1}{\left(n_{j}+\alpha\right)^{2 n-2 k+2}} \prod_{\substack{1 \leqslant m \leqslant k \\ m \neq j}} \frac{1}{\left(n_{m}+\alpha\right)^{2}-\left(n_{j}+\alpha\right)^{2}}\right) .
$$

Now we take each $n_{j}=N$ in turn. Then the sum (15) becomes that

$$
\sum_{N \geqslant 0} \frac{1}{(N+\alpha)^{2 n-2 k+2}} \sum_{j=1}^{k} P(N, j-1) Q(N, k-j),
$$

where the series $\{P(N, i)\}_{i=0}^{\infty}$ and $\{Q(N, i)\}_{i=0}^{\infty}$ are defined in the following manner. Define $P(N, 0)=Q(N, 0) \triangleq 1$, and $Q(N, j)=0, \forall j>N$; If $j>1$ then define $P(N, j-1)$ to be

$$
P(N, j-1)=\sum_{n_{1}>\cdots>n_{j-1}>N} \prod_{i=1}^{j-1} \frac{1}{\left[\left(n_{i}+\alpha\right)^{2}-(N+\alpha)^{2}\right]}
$$

While if $1 \leqslant j<k$ and $k-j \leqslant N$, then define $Q(N, k-j)$ to be

$$
Q(N, k-j)=\sum_{N>n_{j+1}>\cdots>n_{k} \geqslant 0} \prod_{i=j+1}^{k} \frac{1}{\left[\left(n_{i}+\alpha\right)^{2}-(N+\alpha)^{2}\right]} .
$$

In other words, the series $\{P(N, i)\}_{i=0}^{\infty}$ and $\{Q(N, i)\}_{i=0}^{\infty}$ are defined by the following generating functions:

$$
1+P(N, 1) x+P(N, 2) x^{2}+\cdots=\prod_{r>N}\left(1+\frac{x}{(r+\alpha)^{2}-(N+\alpha)^{2}}\right),
$$

and

$$
1+Q(N, 1) x+Q(N, 2) x^{2}+\cdots=\prod_{0 \leqslant r<N}\left(1+\frac{x}{(r+\alpha)^{2}-(N+\alpha)^{2}}\right) .
$$


Now the second sum $\sum_{j=1}^{k} P(N, j-1) Q(N, k-j)$ in $(16)$ is the coefficients of $x^{k-1}$ in the power series

$$
\left[1+P(N, 1) x+P(N, 2) x^{2}+\cdots\right] \cdot\left[1+Q(N, 1) x+Q(N, 2) x^{2}+\cdots\right],
$$

i.e.,

$$
\sum_{j=1}^{k} P(N, j-1) Q(N, k-j)=a_{k-1}^{(N)},
$$

where $a_{k-1}^{(N)}$ is given by $(1)$.

Therefore, the sum (16) is

$$
\sum_{N \geqslant 0} \frac{a_{k-1}^{(N)}}{(N+\alpha)^{2 n-2 k+2}}
$$

which gives the identity (2).

Proof of Theorem 3. Consider the infinite product

$$
\prod_{r=0}^{\infty}\left(1+\frac{x}{(r+\alpha)^{2}-y^{2}}\right)
$$

where $y$ is a complex number with $y \notin\{\alpha, 1+\alpha, 2+\alpha, \cdots\}$.

By Lemma 7, we have

$$
\begin{aligned}
& \prod_{r=0}^{\infty}\left(1+\frac{x}{(r+\alpha)^{2}-y^{2}}\right) . \\
= & \frac{\alpha^{2}-y^{2}+x}{\alpha^{2}-y^{2}} \prod_{r=1}^{\infty} \frac{\left(r+\alpha+\sqrt{y^{2}-x}\right)\left(r+\alpha-\sqrt{y^{2}-x}\right)}{(r+\alpha+y)(r+\alpha-y)} \\
= & \frac{\alpha^{2}-y^{2}+x}{\alpha^{2}-y^{2}} \frac{\Gamma(1+\alpha+y) \Gamma(1+\alpha-y)}{\Gamma\left(1+\alpha+\sqrt{y^{2}-x}\right) \Gamma\left(1+\alpha-\sqrt{y^{2}-x}\right)} \\
= & \frac{\Gamma(\alpha+y) \Gamma(\alpha-y)}{\Gamma\left(\alpha+\sqrt{y^{2}-x}\right) \Gamma\left(\alpha-\sqrt{y^{2}-x}\right)} .
\end{aligned}
$$

It follows that

$$
\prod_{\substack{r=0 \\ r \neq N}}^{\infty}\left(1+\frac{x}{(r+\alpha)^{2}-y^{2}}\right)=\frac{\Gamma(\alpha+y) \Gamma(\alpha-y)}{\Gamma\left(\alpha+\sqrt{y^{2}-x}\right) \Gamma\left(\alpha-\sqrt{y^{2}-x}\right)} \times \frac{(N+\alpha)^{2}-y^{2}}{(N+\alpha)^{2}-y^{2}+x}
$$

By the residue formula of the Gamma function, we have

$$
\lim _{y \rightarrow N+\alpha} \Gamma(\alpha-y)(N+\alpha-y)=\lim _{s \rightarrow-N} \Gamma(s)(s+N)=\frac{(-1)^{N}}{N !} .
$$

Now on both sides of (18) we put $y \rightarrow N+\alpha$. Then we get the required identity in Theorem 3. 
Proof of Corollary 5 and Corollary 6. In Corollary 4, we take $\alpha=1$ and $\alpha=1 / 2$, respectively. By Lemma 8, we have

$$
\begin{gathered}
\frac{1}{\Gamma\left(1+\sqrt{(N+1)^{2}-x}\right) \Gamma\left(1-\sqrt{(N+1)^{2}-x}\right)}=\frac{\sin \left(\pi \sqrt{(N+1)^{2}-x}\right)}{\pi \sqrt{(N+1)^{2}-x}}, \\
\frac{1}{\Gamma\left(\frac{1}{2}+\sqrt{\left(N+\frac{1}{2}\right)^{2}-x}\right) \Gamma\left(\frac{1}{2}-\sqrt{\left(N+\frac{1}{2}\right)^{2}-x}\right)}=\frac{1}{\pi} \cos \left(\pi \sqrt{\left(N+\frac{1}{2}\right)^{2}-x}\right) .
\end{gathered}
$$

It is easy to verify that

$$
\begin{aligned}
& \left.\left(\frac{\sin \left(\pi \sqrt{(N+1)^{2}-x}\right)}{\pi \sqrt{(N+1)^{2}-x}}\right)^{(k)}\right|_{x=0}=\left.(-1)^{k} \pi^{2 k}\left(\frac{\sin \sqrt{x}}{\sqrt{x}}\right)^{(k)}\right|_{x=(N \pi+\pi)^{2}} \\
= & \left.2(-1)^{k+1} \pi^{2 k}(\cos \sqrt{x})^{(k+1)}\right|_{x=(N \pi+\pi)^{2}} \\
& \left.\left(\cos \left(\pi \sqrt{\left(N+\frac{1}{2}\right)^{2}-x}\right)\right)^{(k)}\right|_{x=0}=\left.(-1)^{k} \pi^{2 k}(\cos \sqrt{x})^{(k)}\right|_{x=(N \pi+\pi / 2)^{2}} .
\end{aligned}
$$

Substitute the right-hand side of (19) into the right-hand side of the identity in Corollary 4 , then we get the identity (3). Similarly, we can prove (4).

To prove Corollary 6, we use Lemma 11. It is deduced from (12) that

$$
\begin{aligned}
& \left.(\cos \sqrt{x})^{(k+1)}\right|_{x=(N \pi+\pi)^{2}} \\
& =\frac{(-1)^{(N+k+1)}}{(2 \pi)^{2 k}} \sum_{j=0}^{\left\lfloor\frac{k-1}{2}\right\rfloor} \frac{(-1)^{j}(2 k-1-2 j) !}{(2 j+1) !(k-2 j-1) !} \frac{(2 \pi)^{2 j}}{(N+1)^{2 k-2 j}}, \\
& \left.(\cos \sqrt{x})^{(k)}\right|_{x=(N \pi+\pi / 2)^{2}} \\
& =\frac{(-1)^{(N+k)}}{(2 \pi)^{2 k-1}} \sum_{j=0}^{\left\lfloor\frac{k-1}{2}\right\rfloor} \frac{(-1)^{j}(2 k-2-2 j) !}{(2 j) !(k-2 j-1) !} \frac{(2 \pi)^{2 j}}{\left(N+\frac{1}{2}\right)^{2 k-1-2 j}} .
\end{aligned}
$$

Substitute (21) and (22) into (3) and (4) respectively, then we will get the desired identities (5) and (6). 


\section{Generalizations}

Similar to the definition of $E(2 n, k ; \alpha)$, we can define a number $E(m n, k ; \alpha)$ as follows. Let $m, n$ and $k$ be positive integers with $m \geqslant 2$ and $n \geqslant k$. Then define the number $E(m n, k ; \alpha)$ to be

$$
E(m n, k ; \alpha)=\sum_{\substack{j_{1}+\cdots+j_{k}=n \\ j_{1}, j_{2}, \cdots, j_{k} \geqslant 1}} \zeta\left(m j_{1}, m j_{2}, \cdots, m j_{k} ; \alpha\right) .
$$

If we check the proofs of Theorem 2 and Theorem 3 again, we may find that the theorems can be generalized in the following way.

Theorem 12. Let $\alpha$ be a complex number with $\alpha \notin\{0,-1,-2, \cdots\}$ and let $N \geqslant 0$ denote an arbitrary integer. Define a function $f_{N}(x)$ by

$$
f_{N}(x)=\prod_{\substack{r=0 \\ r \neq N}}^{\infty}\left(1+\frac{x}{(r+\alpha)^{m}-(N+\alpha)^{m}}\right)
$$

Then for positive integers $n$ and $k$ (suppose $n \geqslant k$ ), we have

$$
E(m n, k ; \alpha)=\frac{1}{(k-1) !} \sum_{N=0}^{\infty} \frac{\left.\left[f_{N}(x)\right]^{(k-1)}\right|_{x=0}}{(N+\alpha)^{m n-m k+m}} .
$$

Moreover, the function $f_{N}(x)$ can be represented by the Gamma function, i.e.,

$$
f_{N}(x)=\frac{(-1)^{N} m(N+\alpha)^{m-1}}{N !} \cdot \frac{1}{x} \cdot \frac{\prod_{i=1}^{m-1} \Gamma\left(\alpha-\theta_{i}(N+\alpha)\right)}{\prod_{i=1}^{m} \Gamma\left(\alpha-\theta_{i} \sqrt[m]{(N+\alpha)^{m}-x}\right)},
$$

where $\theta_{1}, \theta_{2}, \cdots, \theta_{m}(\triangleq 1)$ are all the pairwise different roots of $Z^{m}=1$ in $\mathbb{C}$.

\section{Acknowledgements}

The authors would like to thank the referee for helpful suggestions.

\section{References}

[1] H. Gangl, M. Kaneko, D. Zagier. Double zeta values and modular forms. In Siegfried (Ed.), Automorphic Forms and Zeta Functions. In Memory of Tsuneo Arakawa, Proc. of the Conf., Rikkyo University, Tokyo, Japan, 4-7 September 2004, pages 71-106. World Scientific Böherer, Hackensack, NJ, 2006.

[2] A. Granville. A decomposition of Riemann's zeta function. In Analytic Number Theory, Volume 247 of London Math. Soc. Lecture Note Ser., pages 95-101. Cambridge University Press, 1997. 
[3] M. E. Hoffman. Multiple harmonic series. Pacific J. Math, 152(2): 275-290, 1992.

[4] M. E. Hoffman, C. Moen. Sums of triple harmonic series. J. Number Theory, 60(2): 329-331, 1996.

[5] M. E. Hoffman. On multiple zeta values of even arguments. arXiv:1205.7051

[6] M. Igarashi. On generalizations of the sum formula for multiple zeta values. arXiv: 1110.4875

[7] M. Kaneko. Introduction to multiple zeta values, (in Japanese). In Algebraic number theory and related topics. (Kyoto, 1998), pages 50-68. Sūrikaisekikenkyūsho Kōkyūroku 1097, 1999.

[8] Y. Liu. Cylinder functions (in Chinese), National Defence Industrial Press, Bejing, 1983.

[9] C. Markett. Triple sums and the Riemann zeta function. J. Number Theory, 48(2): 113-132, 1994.

[10] R. Remmert. Classical topics in complex function theory, GTM172, Springer, 2013.

[11] Z. Y. Shen, T. X. Cai. Some identities for multiple zeta values. J. Number Theory, 132: 314-323, 2012.

[12] J. Q. Zhao, Sum Formula of Multiple Hurwitz-Zeta Values. arXiv:1207.2368 